\title{
Limitations of LEED-Structure Determination of Reconstructed Crystal Surfaces
}

\author{
H. Jagodzinski \\ Institut für Kristallographie und Mineralogie der Universität München
}

Z. Naturforsch. 37 a, 1103-1118 (1982); received August 25, 1982

Dedicated to Prof. G. Hildebrandt on the occasion of his 60th birthday

\begin{abstract}
Although a strictly ordered reconstructed crystal surface may have the two-dimensional symmetry prescribed by the bulk (two-dimensional subsymmetry of the 3-dimensional space group), it belongs more frequently to a subgroup. In the LEED-pattern a lower symmetry of the translation group can easily be detected on account of the superstructure reflections observed. In this case anti-phase domains can hardly be avoided. An even more difficult situation arises if the symmetry of the point group is violated, although the symmetry of the diffraction pattern is not altered at the end of the reconstruction. Twin domains without changes of lattice constants have to be taken into account.

Dynamical scattering of anti-phase domains is calculated by applying the "Cluster embedded chain"-method for various distributions of domains. It is shown that the incoherent superposition of scattering amplitudes, normally applied in LEED-calculations may lead to serious errors in structure determination. This effect is even more pronounced for twin domains. As an example we discuss the reconstruction of the (001)-surface of $\mathrm{Si}$ and $\mathrm{Ge}$, and show that the wellknown $(2 \times 1)$-structure is an averaged structure only, consisting of anti-phase domains with the $(4 \times 2)$-structure. It may be concluded therefrom that none of the present model structures of this reconstruction agrees with this experimental observation.
\end{abstract}

\section{Introduction}

It is generally accepted that structure determinations of crystal surfaces with the aid of low energy electron diffraction (LEED) demand a dynamical solution of the diffraction problem. The agreement between observed and calculated intensities of the diffracted electron beams may be very good; e.g. Noonan and Davis [1] could explain the surface structure of $\mathrm{Cu}(100)$ for 4 beams with a reliability factor after Zanazzi and Jona [2] of 0.044. Other structure determinations do not agree as well, especially in cases, where a reconstruction involves a change of lattice constants. Disagreement between observed and calculated intensities may be due to an incorrect scattering potential of the atoms, or to the non-periodic absorption potential entering the computer program; but as to my knowledge of diffraction problems, major uncertainties result from the assumption of a strictly ordered surface, which need not be realized necessarily. Although domain structures are sometimes considered, all structure determinations of reconstructed surfaces

Reprint requests to Prof. Dr. H. Jagodzinski, Mineralogische Staatssammlung, Theresienstr. 41, D-8000 München 2. are based on the principle of interpreting the diffraction pattern in terms of an incoherent superposition of domain scattering. Limitations of this assumption will be discussed in this paper, and it will be shown that in certain cases there exist considerable deviations between calculated and observed intensities. Therefore a careful study of the disorder problem of any crystal surface is necessary, before the final structure determination can be done. Only in this way a reliable description of the surface structure can be given. Much confusion due to incorrect models of reconstructed crystal surfaces could have been avoided if the authors had been familiar with the influence of disorder on the integrated intensities of the scattered beams.

Obviously the relatively high background scattering in LEED-patterns hampers the analysis of disorder phenomena considerably. Another difficulty is caused by the limited resolution power of the diffraction unit. It should be pointed out that structure determinations are not rare, where diffuse scattering or weak beams are indeed observed, but not considered for the solution of the problem. It is the aim of this paper to show, how these phenomena have to be regarded in structure determination.

0340-4811 / 82 / 1000-1103 $\$ 01.30 / 0$. - Please order a reprint rather than making your own copy. 


\section{Domains and Symmetry in Reconstructions}

Since all structure investigations of crystal surfaces use low indexed surfaces of high symmetry, some comments on symmetry should be given. Obviously the termination of the bulk crystal by a low indexed plane reduces the symmetry to a plane group containing all symmetry elements which are nct destroyed by that plane; they are: mirror planes and rotation axes vertical to the surface, glide planes with a glide translation parallel to the surface, and all translations parallel to the surface. Therefore the symmetry of the crystal is reduced to one of the seventeen plane groups. For a general surface four types of reconstruction may be discussed:

1. Reconstructions fully obeying all symmetry operations of the said 2-dimensional group. No example may be given so far, except for displacements of the topmost layers.

2. Reconstructions with a lower symmetry of the point group, but without changing the translation group.

3. Reconstructions with a superlattice. (Change of the translation group).

4. Reconstructions involving a change of the translation gro up and the point group.

In literature only the last two examples $(3,4)$ are generally described as reconstruction; they may easily be detected, because superstructure reflections of a unit cell with basis vectors $m \boldsymbol{a}, n \boldsymbol{b}$ appear in the diffraction pattern, generally called a $(m \times n)$ superstructure. But there are difficulties in differentiating between 1 and 2 , or 3 and 4 as well.

Since the bulk crystal determines a maximum symmetry, any reconstruction must be a subgroup of the 2-dimensional group. The fact that in most cases the full symmetry of the said group is observed can only be explained by assuming domains generated by the lost symmetry elements of the full symmetry group (plane group). If the abundance of symmetrically equivalent domains is equal, the full symmetry of the crystal surface is realized in the statistical sense only. In accordance with the definitions given for three-dimensional crystals, let us introduce the following names for the various types:

Type 1: No domains (primitive reconstruction), Type 2: twin domains of $m$-, 2-, 3-, 6-symmetry,
Type 3 : anti-phase domains,

Type 4: anti-phase twin domains.

Domains necessitate boundaries generating an unfavourable boundary energy. Whether or not this energy may be avoided is a question of mobility of the boundaries themselves. Their movement may be hampered by the activation energy necessary to start migration. Furthermore it is well known that lattice defects at the surface may stabilize the boundary, thus preventing the crystal surface from becoming a single domain. Consequently the existence of domains is most probable in reconstructions of type 2,3 and 4 .

Analyses of domains with the aid of diffraction methods is facilitated if the average domain sizes are sufficiently small, and the boundaries between domains are straight lines, normally following low indexed directions of the lattice. Obviously the case of a single direction is easiest to handle (lamellar domains), since their diffuse scattering is concentrated on lines in the diffraction pattern. Irregularly shaped domains are hardly detected in a distribution of domains of type 1 or 2 . They are hidden in the background of the diffraction pattern if their average size is larger than the corresponding resolution power of the LEED-diffraction unit. Consequently the actual average size of domains should not exceed a few hundred $\AA$, otherwise an influence on the reflection profiles can hardly be detected.

Superstructure reflections are typical for domains of types 3 and 4 . Their line profiles may be used for determining the average domain size. This method may be applied if all superstructure reflections have the same line profile. Other cases necessitate a theoretical model, before solving the problem of the averaged domain sizes. It should be added without proof that domain distributions of type 3 and 4 may easily be differentiated by studying the diffuseness of line profiles of all reflections. If the "host"-reflections are sharp without a clear diffuseness in their immediate neighbourhood, type 3 (antiphase domains) is realized. Type 4 is characterized by an additional diffuseness around the host reflections.

\section{Diffraction Theory of Reconstrueted Surfaces}

Semi-quantitative solutions of the LEED-diffraction problem for disordered systems may be given 
with the aid of a theory published by Jagodzinski, Moritz and Wolf [3]. Their method was called [4] the CEC-Method (Cluster-Embedded-Chain method) on account of the fact that the crystal surface is subdivided into chains (perpendicular to the surface) which are embedded in a certain surrounding of limited size (cluster). The length of this chain is determined by the penetration length of the electrons used for diffraction. To calculate the wave function of the scattered electron, the contribution $F_{\nu}\left(\boldsymbol{k}, \boldsymbol{k}^{\prime}\right)$ of the chain $v$ to the wave function is used. For a practical solution of this problem a laborious procedure has been proposed by Moritz, Jagodzinski, and Wolf [5]. Nevertheless the method may be applied semi-quantitatively without knowing the $\boldsymbol{F}_{\boldsymbol{v}}\left(\boldsymbol{k}, \boldsymbol{k}^{\prime}\right)$ of the chains explicitly. The reason of this effective approximation is a more rapid convergence of the multiple scattering problem, when compared with a normal cluster method. This statement may be understood by considering all last scattering events into the direction $\boldsymbol{k}$ originating from the atoms of any chain perpendicular to the surface. Its contribution to the wave function of the scattered electron is determined by the primary beam, and all last but one scattering events originating from the surrounding atoms (the atoms of the said chain are irradiated by the primary beam and the surrounding atoms!). Now, the latter influence may be developed into a series of contributions of nearest, next nearest etc. neighbours. In the zeroth approximation no neighbours, in the first one nearest neighbours etc. are taken into account. Since their influence is determined by spherical waves, diminishing proportional to the inverse of their distance, and absorbtion is very large, the influence of far neighbours will be small. Therefore it seems to be justified to calculate the influence of neighbouring atoms within a given cluster accurately and to take account of the further surrounding by an averaged periodic one. (Naturally a more accurate, but tedious procedure would be obtained by extending the size of the "cluster".) This method is advantageous because each chain perpendicular to the surface appears once as central chain, in contrast to a normal cluster calculation, where the chains may be subdivided into "inner" and "boundary" chains. Since chains at the boundary are irradiated by the primary beam, by atoms inside, and a large number of atoms outside the cluster, the convergence of any cluster method must be very bad if multiple scattering is important. This is stressed by the fact that the surrounding atoms contain nearest neighbours. The latter does not occur with the CEC-method.

Another advantage of this method shall be discussed very briefly. A simple problem of structural surface crystallography is given by a single overlayer with a structure differing from the bulk. Now, let us assume that only two types of chains perpendicular to the surface exist in principle. The topology of these chains be determined by nearest neighbour interactions within them. The influence of the neighbouring chains (next neighbours) on the structure of the central chain shall be given by displacements of the atoms without any change of their topology (no voids or interchange of two different atoms). Now, in a statistical arrangement of chains we have as many chains differing in atomic positions as there are different configurations of nearest neighbours. If the coordination number is 4 , there are $n=2^{4}=16$ configurations, some of them may be related by symmetry. Since multiple scattering depends on the arrangement of neighbours, we have to introduce 16 different contributions for each central chain in each different configuration of its neighbours. Obviously, all structural changes within the central chain (due to its neighbours) may be included into to the said contribution to scattering. Consequently we define

$$
F_{\nu}\left(\boldsymbol{k}, \boldsymbol{k}^{\prime}\right)
$$

describing the contribution of the central chain in the configuration $v$ - to scattering into the direction $\boldsymbol{k}\left(\boldsymbol{k}^{\prime}=\right.$ direction of incidence). For the sake of simplicity we use for both chains one index only. Hence, $v=1, \ldots, n$ refers to configurations with the first chain, $v=n+1, \ldots, 2 n$ to configurations with the second chains as central ones.

It is shown in Sect. 1 of the appendix that the problem of multiple scattering may be solved approximately for a random arrangement of chains. The following two terms for sharp reflections (beams, $h, k$ in (1 a)) and diffuse reflections given approximately in (1 b) are typical for any disorder problem. (Both equations are derived in Sect. 1 of the Appendix.)

$$
\begin{aligned}
I_{\mathrm{S}}\left(\boldsymbol{k}, \boldsymbol{k}^{\prime}\right) & =R^{2}\left|\sum_{v} p_{\nu} F_{v}\left(\boldsymbol{k}, \boldsymbol{k}^{\prime}\right)\right|^{2}=R^{2}|\bar{F}|^{2},(1 \\
I_{\mathrm{d}}\left(\boldsymbol{k}, \boldsymbol{k}^{\prime}\right) & \left.=\left.N_{\mathrm{q}} N_{\mathrm{b}}\left|\sum_{v} p_{v}\right| F_{v}\left(\boldsymbol{k}, \boldsymbol{k}^{\prime}\right)\right|^{2}-|\bar{F}|^{2}\right], \\
& =N_{\mathrm{a}} N_{\mathrm{b}}\left[\left.\bar{F}\right|^{2}-|\bar{F}|^{2}\right],
\end{aligned}
$$




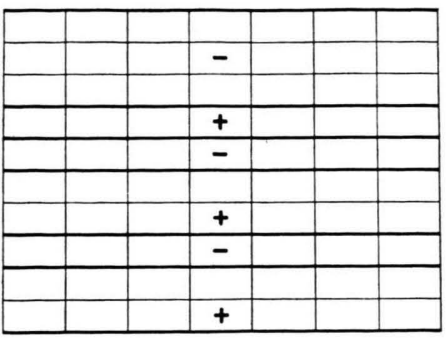

Fig. 1. Lamellar anti-phase domains with a nearly random distribution function; the two possible positions are marked by + and - . The width of domains corresponds to the number of unit cells involved.

where $p_{v}$ is the a-priori probability of the configuration $v$, and

$$
R=\frac{\sin \pi N_{\mathrm{a}} h}{\sin \pi h} \frac{\sin \pi N_{\mathrm{b}} k}{\sin \pi k}
$$

is the well known Laue-factor, which may be replaced by a sum of $\delta$-functions. $N_{\mathrm{a}}, N_{\mathrm{b}}$ are the numbers of unit cells in the $\boldsymbol{a}, \boldsymbol{b}$-direction, while $h$, $k$ are the components of vectors in reciprocal space (continuous variables).

Equation (1a) gives the sharp reflections modulated by an averaged dynamical structure factor corresponding to an averaged periodic lattice. As pointed out in the Appendix, $(1 \mathrm{~b})$ is approximately valid for a random distribution of cluster embedded chains only. Short range order concentrates the diffuse scattering into diffuse peaks, whose positions may be displaced from their ideal ones, as defined by the corresponding long range order.

Let us explain the two formulae in a very simple case, frequently realized in practical structure determination. We consider a surface of a cubic crystal with a primitive upper layer. The nonreconstructed ordered surface only has one chaintype embedded in equal surroundings (clusters). The reconstruction of a $(1 \times 2)$-structure is observed. If the ordering process propagates much more rapidly into the $\boldsymbol{b}$-direction (where the formation of the superstructure takes place), then, in the first stage of reconstruction, a random distribution of ordered chains is realized, corresponding to a distribution of lamellar anti-phase domains (Figure 1). In terms of the CEC-method two types of chains are strictly ordered (alternating sequence) in the $\boldsymbol{b}$-direction. Their contribution to scattering may be treated as described in the Appendix. Since two chains in a different surrounding within the ordered array are realized only, we get a kind of structure factor of the one-dimensional array of chains, (row), which is called $F_{0}\left(\boldsymbol{k}, \boldsymbol{k}^{\prime}\right)$ (Appendix 3 ).

In this particular case three solutions my be given:

1. The statistical distribution,

2. the periodic distribution of domains,

3. the single domain (ideal structure).

We solve these problems for the simple case of multiple scattering, where only nearest neighbours are taken into account. Then 8 types of cluster embedded chains have to be considered as given in Table 1, they may be subdivided into 4 equivalent pairs $1-4$.

The last column in Table 1 gives the a prioriprobabilities of the corresponding central chain to be embedded in the corresponding next neighbours if $1-\alpha$ is the probability of equal neighbours. The $\Delta F_{v}\left(\boldsymbol{k}, \boldsymbol{k}^{\prime}\right)$ represent the dynamical correction for $F_{0}\left(\boldsymbol{k}, \boldsymbol{k}^{\prime}\right)$. It is shown in Sect. 3 of the Appendix that they are not small, when compared to the pseudo-kinematic contribution to the wave function $F_{0}$ (structure amplitude). It should be pointed out that the index $k^{\prime}$ refers to a doubled $\boldsymbol{b}$-axis, $\boldsymbol{b}^{\prime}-\mathbf{2} \boldsymbol{b}$, $k^{\prime}=2 k$. (In LEED-papers often new unit-cells for superstructures are introduced without changing the basis vectors and the components of the reciprocal lattice. Then the superstructure reflections are given by fractional indices, in this case we have $k=k^{\prime} \mid 2$, and beams $k^{\prime}=$ odd correspond to half

Table 1. Dynamical structure amplitudes of chains in a different surrounding and their probabilities. Compare (A 13), (A 13a), and section 4 in the Appendix. The factor $1 / 2$-corresponding to the a priori-probabilities of central chains has been omitted.

\begin{tabular}{llllll}
\hline Nr. & Symb. & Dynamic structure factor & Symb. & Dynamic structure factor & Probabilities \\
\hline $\mathbf{1}$ & $\mathbf{1 1 1}$ & $F_{0}\left(\boldsymbol{k}, \boldsymbol{k}^{\prime}\right)+\Delta F_{1}\left(\boldsymbol{k}, \boldsymbol{k}^{\prime}\right)$ & 222 & {$\left[F_{0}\left(\boldsymbol{k}, \boldsymbol{k}^{\prime}\right)+\Delta F_{1}\left(\boldsymbol{k}, \boldsymbol{k}^{\prime}\right)\right] e^{\pi i k}$} & $(1-\alpha)^{2}$ \\
$\mathbf{2}$ & $\mathbf{1 1 2}$ & $F_{0}\left(\boldsymbol{k}, \boldsymbol{k}^{\prime}\right)+\Delta F_{2}\left(\boldsymbol{k}, \boldsymbol{k}^{\prime}\right)$ & 221 & {$\left[F_{0}\left(\boldsymbol{k}, \boldsymbol{k}^{\prime}\right)+\Delta F_{2}\left(\boldsymbol{k}, \boldsymbol{k}^{\prime}\right)\right] e^{\pi i k}$} & $\alpha(1-\alpha)$ \\
3 & 211 & $F_{0}\left(\boldsymbol{k}, \boldsymbol{k}^{\prime}\right)+\Delta F_{3}\left(\boldsymbol{k}, \boldsymbol{k}^{\prime}\right)$ & $\mathbf{1 2 2}$ & {$\left[F_{0}\left(\boldsymbol{k}, \boldsymbol{k}^{\prime}\right)+\Delta F_{3}\left(\boldsymbol{k}, \boldsymbol{k}^{\prime}\right)\right] e^{\pi i k}$} & $\alpha(1-\alpha)$ \\
$\mathbf{4}$ & $\mathbf{2 1 2}$ & $F_{\mathbf{0}}\left(\boldsymbol{k}, \boldsymbol{k}^{\prime}\right)+\Delta F_{4}\left(\boldsymbol{k}, \boldsymbol{k}^{\prime}\right)$ & $\mathbf{1 2 1}$ & {$\left[F_{0}\left(\boldsymbol{k}, \boldsymbol{k}^{\prime}\right)+\Delta F_{4}\left(\boldsymbol{k}, \boldsymbol{k}^{\prime}\right)\right] e^{\pi i k}$} & $\alpha^{2}$ \\
\hline
\end{tabular}


order beams). The evaluation of (1a, b) is given in Appendix 4. We get from (A 18) and (A 19):

a) $k^{\prime}=2 n(k=n)$ :

$$
\begin{aligned}
|\bar{F}|^{2}=\left|F_{0}\right|^{2} & +F_{0}\left[(1-\alpha)^{2} \Delta F_{1} *+\alpha(1-\alpha)\left(\Delta F_{2} *+\Delta F_{3} *\right)+\alpha^{2} \Delta F_{4} *\right] \\
& +F_{0} *\left[(1-\alpha)^{2} \Delta F_{1}+\alpha(1-\alpha)\left(\Delta F_{2}+\Delta F_{3}\right)+\alpha^{2} \Delta F_{4}\right] \\
& +(1-\alpha)^{4}\left|\Delta F_{1}\right|^{2}+\alpha^{2}(1-\alpha)^{2}\left|\Delta F_{2}+\Delta F_{3}\right|^{2}+\alpha^{4}\left|\Delta F_{4}\right|^{2} \\
& +\alpha(1-\alpha)^{3}\left[\Delta F_{1}\left(\Delta F_{2} *+\Delta F_{3} *\right)+\Delta F_{1} *\left(\Delta F_{2}+\Delta F_{3}\right)\right] \\
& +\alpha^{2}(1-\alpha)^{2}\left[\Delta F_{1} \Delta F_{4} *+\Delta F_{1} * \Delta F_{4}\right] \\
& +\alpha^{3}(1-\alpha)\left[\Delta F_{4}\left(\Delta F_{2} *+\Delta F_{3} *\right)+\Delta F_{4} *\left(\Delta F_{2}+\Delta F_{3}\right)\right] . \\
\left.\left|\overline{\left.F\right|^{2}}=\right| F_{0}\right|^{2} & +F_{0}\left[(1-\alpha)^{2} \Delta F_{1} * \alpha(1-\alpha)\left(\Delta F_{2} *+\Delta F_{3} *\right)+\alpha^{2} \Delta F_{4} *\right] \\
& +F_{0} *\left[(1-\alpha)^{2} \Delta F_{1}+\alpha(1-\alpha)\left(\Delta F_{2}+\Delta F_{3}\right)+\alpha^{2} \Delta F_{4}\right] \\
& +(1-\alpha)^{2}\left|\Delta F_{1}\right|^{2}+\alpha(1-\alpha)\left(\left|\Delta F_{2}\right|^{2}+\left|\Delta F_{3}\right|^{2}\right)+\alpha^{2}\left|\Delta F_{4}\right|^{2} .
\end{aligned}
$$

Introducing (2a), into (1a), and (2b) into (A9) we get for $k^{\prime}=2 n(k=n)$

$$
\begin{aligned}
I_{\mathrm{S}}\left(\boldsymbol{k}, \boldsymbol{k}^{\prime}\right)= & R^{2}|\bar{F}|^{2}, \text { valid for all } \alpha, \\
I_{\mathrm{d}}\left(\boldsymbol{k}, \boldsymbol{k}^{\prime}\right)= & N_{\mathrm{a}} R_{\mathrm{b}}^{\prime 2}\left\{2 \alpha(1-\alpha)^{2}(1-\alpha / 2)\left|\Delta F_{1}\right|^{2}+\alpha^{2}(1-\alpha)^{2}\left(1+\alpha^{2} /(1-\alpha)\right)\left(\left|\Delta F_{2}\right|^{2}+\left|\Delta F_{3}\right|^{2}\right)\right. \\
& +\alpha^{2}(1-\alpha)^{2}\left|\Delta F_{4}\right|^{2}-\alpha(1-\alpha)^{3}\left[\Delta F_{1}\left(\Delta F_{2} *+\Delta F_{3} *\right)+\Delta F_{1} *\left(\Delta F_{2}+\Delta F_{3}\right)\right] \\
& -\alpha^{2}(1-\alpha)^{2}\left[\Delta F_{1} \Delta F_{4} *+\Delta F_{1} * \Delta F_{4}+\Delta F_{2} * \Delta F_{3}+\Delta F_{2} \Delta F_{3} *\right] \\
& \left.-\alpha^{3}(1-\alpha)\left[\Delta F_{4}\left(\Delta F_{2} *+\Delta F_{3} *\right)+\Delta F_{4} *\left(\Delta F_{2}+\Delta F_{3}\right)\right]\right\}
\end{aligned}
$$

with $R_{\mathrm{b}}{ }^{\prime}=\sin \pi N_{\mathrm{b}} k^{\prime} / \sin \pi k$.

For $\alpha \rightarrow 0$ (3a) reduces to $I_{\mathrm{s}}=R^{2}\left|F_{0}+\Delta F_{1}\right|^{2}$ (scattering of a single domain), but for non-vanishing small values of $\alpha(3 \mathrm{a})$ becomes

$$
I_{\mathrm{s}}=R^{2}\left|F_{0}+(1-\alpha)^{2} \Delta F_{1}+\alpha(1-\alpha)\left(\Delta F_{2}+\Delta F_{3}\right)\right|^{2} .
$$

This means that there is a considerable influence on intensities of sharp reflections, vanishing only if $\Delta F_{v}=0$. This yields the general law that the invariability of the kinematic scattering with $\alpha$ is violated by the dynamical scattering even for the sharp reflections. Since the incoherent superposition of the scattering from two domains is identical with the scattering of a single domain $\left|F_{0}+\Delta F_{1}\right|^{2}$, structure determination without considering the disorder parameter explicitly is becoming increasingly doubtful.

Since $1 / \alpha$ is the average number of cells along the $\boldsymbol{a}$-direction, the average domain length becomes $100 \AA$ with $\alpha=0,05$ and $a=5 \AA$ for example. Consequently the diffraction effects of these domains can not easily be detected. The correction introduced by $\alpha, \Delta F_{2}$ and $\Delta F_{3}$ in $(3 \mathrm{c})$ is about $10 \%$, if $F_{0}$ and $\Delta F_{2}, \Delta F_{3}$ have the same order of magnitude (compare Appendix 4). A similar discussion of $(3 \mathrm{~b})$ shows that the diffuse scattering vanishes for $\alpha=0$. If $\alpha=0,05$ there is again a diffuse scattering, whose integrated intensities have the same order of magnitude as the loss in intensity of sharp reflections. This is in contrast to a kinematic calculation of diffuse scattering, where it vanishes for $k^{\prime}=2 n$.

b) $k^{\prime}=2 n+1\left(k=\frac{2 n+1}{2}\right)$ :

If $k^{\prime}$ is odd $F$ vanishes, since all pairs of clusters given in Table 1 have equal a-priori probabilities and one of them has a phase factor $e^{i \pi k^{\prime}}=-1$, consequently $\bar{F}$ becomes zero. According to (3a) the sharp reflections vanish in agreement with a kinematic calculation of intensities.

Following (A9) the diffuse intensity becomes $I_{\mathrm{d}}=N_{\mathrm{a}} R_{\mathrm{b}} 2 \sqrt{|F|^{2}}$, where $\overline{|F|^{2}}$ is given in $(2 \mathrm{~b})$. This intensity is much stronger than the diffuse intensity calculated for $k^{\prime}=2 n$ (Equation $3 \mathrm{~b}$ ). The dynamical correction for diffuse scattering has the same order of magnitude as for the sharp reflections, but the intensity is distributed continuously on a line in reciprocal space perpendicular to $\boldsymbol{b}$ ( $\boldsymbol{a}^{*}$-direction). On account of (A7) the diffuse scattering concentrates into diffuse peaks if $\alpha \neq 0,5$. Hence, (3b) for diffuse scattering is approximately valid 
for $\alpha=0,5$ only, and the whole question has to be reconsidered for periodic distribution functions of domains.

A few comments should be added for domains belonging to type 2 and 4 , defined in Chapter 2 . It may easily be shown that in this case $|\bar{F}|^{2}$ in (1a) refers to an averaged structure of at least two domains. It will be demonstrated below that an incoherent treatment of the diffracted intensities may lead to an incorrect interpretation, even in the kinematical treatment of disordered structures as used in X-ray and Neutron-diffraction.

Let us now discuss the model of the periodic array of anti-phase domains (equal size). In this particular case no clusters (212) and (121) (correlated with $\Delta F_{4}$ ) occur (compare the boundary in Figure 2a). Two structures may be realized, the primitive structure shown in Fig. $2 \mathrm{a}$, and the centred structure according to Fig. $2 \mathrm{~b}$. Introduction of a new vector $\boldsymbol{a}^{\prime}$ (Fig. 2 b) leads to the same distribution statistics, since in both structures lines following $\boldsymbol{a}$, $\boldsymbol{a}^{\prime}$, respectively, connect chains in equal positions. Thus Fig. $2 \mathrm{a}$ describes $a(1 \times 2)$ superstructure, while Fig. 2 b represents a centred $(2 \times 2)$-superstructure if the vectors $\boldsymbol{a}, \boldsymbol{b}$ are used. Introducing a new basis $\boldsymbol{a}^{\prime}=\boldsymbol{a}-\boldsymbol{b}$, and $\boldsymbol{b}^{\prime}=\boldsymbol{a}+\boldsymbol{b}$ we arrive at the normal description of a $(\sqrt{2} \times \sqrt{2})$ superstructure. For the diffraction problem of the structure it is more convenient to stick to the $\boldsymbol{b}^{\prime}$-vector being parallel to the boundary of antiphase domains.

The calculation of structure factors with the aid of the CEC-method is not difficult, but tedious. A general solution for X-ray diffraction valid for any type of domain pairs has been given by Jagodzinski and Penzkofer [6], and may be extended to

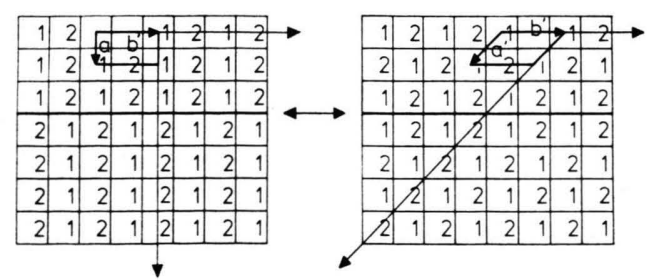

a)

b)
Fig. 2. Boundary of two lamellar anti-phase domains. The doubled $b^{\prime}$-axis $\left(b^{\prime}=2 b\right)$ of the reconstructed surface displaces the two domains by $\boldsymbol{b}^{\prime} / 2$ equivalents with respect to the bulk. a) solution for the primitive arrangement of chains, b) solution for the centred arrangement of chains. Note that both arrangements are equivalent in the two different directions shown in a) and b). include dynamical scattering according to the CEC-method. The procedure will be described in a later paper. The result of this calculation of $A\left(\boldsymbol{k}, \boldsymbol{k}^{\prime}\right)$, with $I\left(\boldsymbol{k}^{\prime}, \boldsymbol{k}^{\prime}\right)=\left|A\left(\boldsymbol{k}, \boldsymbol{k}^{\prime}\right)\right|^{2} \quad$ is: $\quad k^{\prime}=\mathbf{2 n} \quad$ (even) $(k=n)$

$$
\begin{aligned}
A= & R^{\prime}\left(F_{0}+\Delta F_{1}\right) \\
& +R^{\prime \prime}\left\{2 e^{-\pi i h}\left(\cos \pi N_{1} h\right)\left(\Delta F_{2}-\Delta F_{1}\right)\right. \\
& \left.+2 e^{\pi i h}\left(\cos \pi N_{1} h\right)\left(\Delta F_{3}-\Delta F_{1}\right)\right\}
\end{aligned}
$$

where $N_{\mathrm{a}}=2 N_{1} N$, with

$N=$ number of pairs of domains,

$N_{1}=$ number of cells of one domain in the $\boldsymbol{a}$-direction,

and

$$
\begin{aligned}
& R^{\prime}=\frac{\sin \pi N_{\mathrm{a}} h}{\sin \pi h} \frac{\sin \pi N_{\mathrm{b}} k^{\prime}}{\sin \pi k^{\prime}}, \\
& R^{\prime \prime}=\frac{\sin \pi N_{\mathrm{b}} k^{\prime}}{\sin \pi k^{\prime}} \frac{\sin 2 N_{1} N h}{\sin \pi 2 N_{1} h} .
\end{aligned}
$$

Apparently the first term in (4) is equivalent to the dynamical scattering of a single domain and needs no further consideration. The second term has maxima (satellites) for $h=v / 2 N_{1}$ of height $N$, modulated by a structure factor containing dynamical contributions to scattering only.

Two expressions are found for the satellites $v=2 n$, and $v=2 n+1$, respectively $(\neq 0)$ : $v=2 n$,

$$
\begin{aligned}
A= & \frac{\sin \pi N_{\mathrm{b}} k^{\prime}}{\sin \pi k^{\prime}} \frac{\sin \pi N v}{\sin \pi v} \\
& \cdot 2\left\{\left(\Delta F_{2}-\Delta F_{1}\right) \exp \left\{-\pi i v / 2 N_{1}\right\}\right. \\
& \left.\quad+\left(\Delta F_{3}-\Delta F_{1}\right) \exp \left\{+\pi i v / 2 N_{1}\right\}\right\} .
\end{aligned}
$$

As long as $N \gg N_{1}$ there is no important interference between the two terms in (4) unless $v=0$, where a similar correction occurs for the main reflections already discussed for the random case. These main reflections are accompanied by satellites (5) whose amplitudes are regulated by $R^{\prime}, R^{\prime \prime}$, respectively. Unfortunately the conditions $N \gg 2 N_{1}$ cannot be fulfilled in the case of large domain sizes. Here interference effects between the first and second term may take place, but their influence on intensities should be small.

$v=2 n+1$.

The second term in (4) vanishes, and consequently no odd satellites are observed. 
Solution for $k^{\prime}=2 n+1\left(k=\frac{2 n+1}{2}\right):$

If $k^{\prime}$ is odd no main reflections are observed, but two types of satellites occur. $A\left(\boldsymbol{k}, \boldsymbol{k}^{\prime}\right)$ becomes:

$$
\begin{aligned}
A=-\frac{\sin \pi N_{\mathrm{b}} k^{\prime}}{\sin \pi k^{\prime}} & \frac{\sin \pi 2 N_{1} N h}{\sin \pi 2 N_{1} h}\left\{i \frac{1-\cos \pi 2 N_{1} h}{\sin \pi h}\left(F_{0}+\Delta F_{1}\right)\right. \\
& \left.+2 i \exp \left\{i \pi N_{1} h\right\}\left[\left(\Delta F_{2}-\Delta F_{1}\right) e^{-i \pi h} \sin \pi N_{1} h-\left(\Delta F_{3}-\Delta F_{1}\right) e^{i \pi h} \sin \pi N_{1} h\right]\right\} .
\end{aligned}
$$

The maxima $h=v / 2 N_{1}$ are extinguished if $v=2 n$, consequently, odd satellites only are observed. For $v=2 n+1$ (6) may be transformed to

$$
\begin{aligned}
A=-\frac{\sin \pi N_{\mathrm{b}} k^{\prime}}{\sin \pi k^{\prime}} \frac{\sin \pi N v}{\sin \pi v} & \left\{\frac{i}{\sin \pi v / 2 N_{1}}\left(F_{0}+\Delta F_{1}\right)\right. \\
& \left.+2\left[\left(\Delta F_{3}-\Delta F_{1}\right) \exp \left\{i \pi v / 2 N_{1}\right\}-\left(\Delta F_{2}-\Delta F_{1}\right) \exp \left\{-i \pi v / 2 N_{1}\right\}\right]\right\} .
\end{aligned}
$$

Equation (7) may again be discussed for $N \gg N_{1}$ and $N \ll N_{1}$. If $N \gg N_{1}$ (small domain sizes) the interference effect between the two terms in curly brackets in (7) is important, if $N_{1} \gg N$ (a few large domains) the first term dominates, but the side maxima $(v=3,5 \ldots)$ of the first term have to be taken into account also. It should be added that the satellites may become a diffuse peak because of the limited resolution power of the LEED-unit, but it should also be remembered that the intensity distribution of satellites differs appreciably from that given by the side-maxima of the sharp reflection (first term) in (4). Since the resolution power of the experiment and the background scattering determine the number of satellites included into the measurement of integrated intensities, serious errors may occur for structure determination. It should be added that these errors may only be corrected by different scaling factors for the beams $k^{\prime}=2 n+1$ if the antiphase vector is parallel to the surface. Since vertical components may not be excluded (e.g. correlation of a displacement vector with a step, or generation of domains by a screw-axis vertical to the surface etc.), the uncertainty of intensity measurement remains to be effective. The difficulties become even more important if domains of type 2 and 4 have to be considered, since satellites of high intensity also occur in the neighbourhood of sharp reflections, and may give rise to complicated interference effects which will be discussed in a later paper. Figure 3 shows the principical behavior of intensities in reciprocal space for the $(1 \times 2)$ - and the

c $(2 \times 2)$ structure. Both cases differ only in the positions of odd satellites for $k^{\prime}=2 n+1$.

In order to elucidate the correspondence of (3a) with (4), and (2b) with (7), respectively, some remarks should be added. Since $1 / \alpha$ corresponds to $N_{1}$, the second term in (4) becomes small if $\alpha \rightarrow 0$, $N_{1} \rightarrow N_{\mathrm{a}}$, respectively.

Consequently we get

$$
\begin{aligned}
I_{\mathrm{s}}\left(\boldsymbol{k}, \boldsymbol{k}^{\prime}\right) & =\left|A\left(\boldsymbol{k}, \boldsymbol{k}^{\prime}\right)\right|^{2} \\
& =R^{\prime 2}\left|\boldsymbol{F}_{0}\left(\boldsymbol{k}, \boldsymbol{k}^{\prime}\right)+\Delta \boldsymbol{F}_{1}\left(\boldsymbol{k}, \boldsymbol{k}^{\prime}\right)\right|^{2}
\end{aligned}
$$

for $(3 \mathrm{c})$ and (4) as well. With increasing $\alpha$ (decreasing $N_{1}$ ) we have a growing influence of the second term in (4). The maxima $h=v / 2 N_{1}(\approx \alpha / 2 \cdot v)$ with $\nu=0$ should be compared with the terms containing $\Delta F_{v^{\prime}}$ in (3c), while the remaining maxima $h=v / 2 N_{1}$ with $v \neq 0$ should correspond to $I_{\mathrm{d}}$ given in $(3 \mathrm{~b})$. The major difference between these expressions is due to the fact that the maxima given by $v \neq 0$ decrease in intensity with increasing $v$,

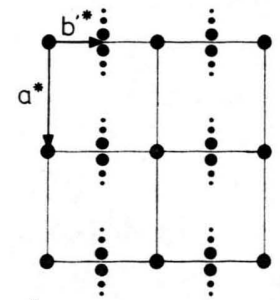

a)

Fig. 3. Reciprocal lattices of the periodical arrangement of domains shown in Figure 2. a) primitiv orthogonal superstructure cell; b) centred orthogonal (or primitive monoclinic) superstructure cell. Note that both pictures are equivalent in two different reciprocal directions. 
while $(3 \mathrm{~b})$ yields a diffuse scattering as determined by all $\Delta F_{v^{\prime}}$, but without pronounced maxima. With increasing $N_{1}$ the more important low order maxima approach the corresponding Bragg-positions and may be regarded as a "diffuse maximum". Diffraction by a non-periodic distribution of domains having a Gaussian distribution of their sizes will differ from (4) in so far as the sharp maxima of the second term have to be replaced by diffuse ones. Their diffuseness increases with growing order of $v$, but the intensity distribution remains essentially the same. Consequently (4) and (7) may be used with this restriction. The mathematical treatment of the influence of various distributions on diffuse scattering has been published by Adlhart [7]. Since his formulae may be extended to include dynamical scattering, we shall not discuss the results here. There are only small differences between both theories affecting the intensities of maxima rather than their positions. Summarizing, the second term in (4) and (7) represents in principle the diffuse reflections caused by any distribution function of lamellar domain sizes with an averaged width of some unit cells at least. Since interference between sharp and diffuse reflections cannot be avoided, structure analyses may be hampered considerably. It should be added without proof that this influence is less important for anti-phase domains than for twin domains, where all basic reflections are accompanied by diffuse ones, whose intensities are not small.

Now we are able to draw some conclusions for structure determination using LEED-data. There are three different possibilities of solving disordered structures:

1. Separation of sharp reflections from diffuse scattering with the aid of high resolution techniques, and solving the structure by means of the averaged structure factor given in (3a), (A5).

2. Using diffuse intensities only, the structure may be solved by applying (A7).

3. With the aid of integrated sharp and diffuse reflections a solution is possible subject to the conditions that the prodominant part of intensity is concentrated into the Bragg-peaks, and interference between the two terms in (4) is negligible. Then the integrated intensities are given by an incoherent summation of the different contributions of domains.
The first method can only be applied if the separation of sharp and diffuse scattering is possible from the experimental point of view. In practice only surface structures with small averaged domain sizes may be solved in this way. But the averaged domain size has to be determined in order to apply the corrections necessary according to $(3 \mathrm{c})$, or (A6), (A 7), respectively.

The second method seems to be possible in any case, unless the diffuse intensities are too small. Consequently, there is a maximum domain size limiting the applicability of this procedure.

The third method seems to be the easiest one from the experimental point of view, but it should be remembered that large domain sizes are necessary. Otherwise a great deal of diffuse intensity is lost, and a bad agreement between calculated and measured intensities results. On the other hand interference between the two terms in (4) may have an important influence on integrated intensities, besides the fact that fluctuations of the areas covered by different domains may also be effective. Consequently a reliable structure determination may be, but needs not necessarily be possible in this way.

Obviously all $(1 \times 1)$-structures determined so far should be correct if no lateral displacement of the upper layers is involved, violating planes of symmetry and rotations perpendicular to the surface. There is also a good chance that surface structures with anti-phase domains only, and with anti-phase vectors parallel to the surface are correct. Appropriate scaling factors for superstructure reflections have to be introduced, and the diffraction pattern must be checked for vanishing diffuse scattering. On the other hand surface structures with twin domains or other defects (e.g. high concentration of terraces) may be incorrect as long as the disorder phenomena have not been considered adequately. Consequently quite a few structure determinations published so far may be incorrect, or even wrong.

\section{Application to Ge and $\mathrm{Si}(001)$}

To illustrate the preceding theoretical calculation we will discuss a schematic picture of the diffraction pattern of a surface published by Olshanetsky, Repinsky and Shklyaev [8]. The diffraction picture shows sharp reflections, and diffuse lines (Fig. 4) 
parallel to $\boldsymbol{a}$ and $\boldsymbol{b}$ (primitive setting of the tetragonal cell), the cubic crystal has the unit cell vectors $\boldsymbol{a}_{\mathrm{c}}=\boldsymbol{a}+\boldsymbol{b}, b_{\mathrm{c}}=\boldsymbol{a}-\boldsymbol{b}$. For the $\mathrm{Si}(001)$ surface the same cell has been reported by Lander and Morrison [9]. Although later papers preferably describe rhombic $(2 \times 1)$-structures, all authors agree in the assumption that domains rotated by $90^{\circ}$ generate the 4-fold symmetry. It will be shown here that all structural models should be incorrect at least as some details are concerned. In order to avoid new confusion let us start with the correct crystallographic setting and symmetry consideration as prescribed by the International Tables for X-ray Crystallography which include two-dimensional space groups. As pointed out above the basis vectors of the 3-dimensional cubic cell $\boldsymbol{a}_{\mathrm{c}}, \boldsymbol{b}_{\mathrm{c}}$ in the (001) plane have to be replaced by

$$
\boldsymbol{a}=\frac{\boldsymbol{a}_{\mathrm{c}}+\boldsymbol{b}_{\mathrm{c}}}{2}, \quad \boldsymbol{b}=\frac{-a_{\mathrm{c}}+b_{\mathrm{c}}}{2} .
$$

This is a primitive tetragonal unit cell at the (001)surface. The symmetry of this plane is p $2 \mathrm{~mm}$, because $4_{1}$ and $4_{3}$ do not belong to the group of 2-dimensional symmetry operations (space group F $4_{1} / \mathrm{d} \overline{3} 2 / \mathrm{m}$ ). Since the glide translation has a vertical component, the d-glide plane in the symmetry of the bulk is lost. The symbol $p$ indicates that the $\boldsymbol{a}, \boldsymbol{b}$-cell is primitive. The fact that diffraction patterns of non-reconstructed (001)-surfaces have a 4 -fold symmetry can be explained by assuming a kind of domain structure (terraces) of the surface: a plane parallel to (001) and displaced by $+c_{\mathrm{c}} / 4$ is a plane which is rotated by $90^{\circ}$ with respect to the original one $\left(4_{1}\right.$-screw axis of the bulk structure). In this way the 4-fold symmetry of the diffraction pattern can be understood if the two orientations occur with equal probabilities. But a completely plane surface should have the lower symmetry p $2 \mathrm{~mm}$. A comparison of the schematic picture of Fig. 4 and the published Si-(100)-pattern of the $(4 \times 2)$-structure reveals that both diffraction patterns are equivalent as already pointed out by Jona, Shih, Jepsen and Marcus [10]. The only difference is that in the case of Ge some of the reflections are diffuse. They are streaks parallel to the unit cell vectors of the tetragonal cell $\boldsymbol{a}, \boldsymbol{b}$. A single domain has the maximum symmetry p $2 \mathrm{~mm}$, but the $b$-axis of the domain has to be doubled, and the new $\boldsymbol{a}^{\prime}$ axis is even four times larger than the original one. This may cause eight

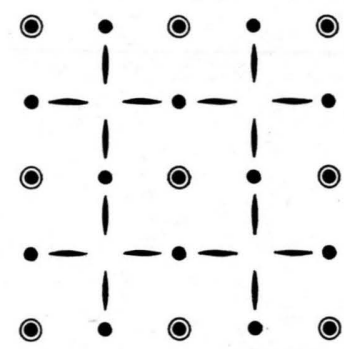

Fig. 4. Schematic drawing of the LEED-direction pattern of Ge (001) after Olshanetsky et al. [8].

anti-phase domains with all combinations of translation vectors $b^{\prime} / 2$, and $\pm \boldsymbol{a}^{\prime} / 4, \boldsymbol{a}^{\prime} / 2$, respectively. But the diffraction picture shows that the latter period is not well established (streaks!). These streaks are rather sharp lines, and apparently they are caused by the boundaries of anti-phase domains. This behaviour is in full agreement with the results given in Sect. 3, compare (2) and (3), respectively. They predict the marked difference between reflections $k^{\prime}=2 n$ and $k^{\prime}=2 n+1$. Since the sharpness of the diffuse lines in the diffraction picture published by Olshanetsky et al. [8] can be compared with that of the basic reflections, the domain must have a considerable length in the $\boldsymbol{b}^{\prime}$-direction, but obviously be short in the $\boldsymbol{a}^{\prime}-(\boldsymbol{a})$ direction, as shown in Figure 1.

But we also have to take into account the twindomains due to the $4_{1}$-screw axis of the bulk crystal, which is responsible for the 4-fold symmetry of the diffraction pattern. If their boundaries were straight lines we would again observe diffuse streaks near the reflections of the basic reciprocal lattice. Apparently this is not true. Consequently there remain only two possibilities:

1. One boundary of the twin domains coincides with the chain direction, the second one may be vertical to it. The diffuse scattering caused by the twin boundaries (diffuse lines) may be hidden by the disorder scattering of the anti-phase domains. In any case there is no extinction rule for twin boundaries. Consequently diffuse lines should also be observed for $k^{\prime}=2 n$. This is not true (Fig. 4) and therefore the domains must be large, as shown in Figure $\mathbf{5}$.

2. The twin domains are smaller, but their boundaries have an irregular shape (Fig. 5 b), such that the diffuse scattering of the domain boundaries is no longer concentrated into diffuse lines. The dif- 


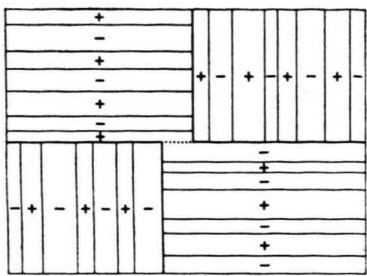

a)

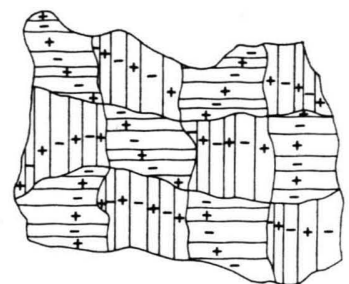

b)
Fig. 5. Two possible distributions for twin and anti-phase domains in Ge and Si. a) Large twin domains with boundaries parallel to the anti-phase boundaries; b) Irregularly shaped smaller twin domains.

fuseness spreads within reciprocal space and may easily be overlooked as long as no broadening of reflection profiles is observed.

Now we have to derive the symmetry of a single domain consisting of a few chains. As mentioned above their maximum symmetry is $p 2 \mathrm{~mm}$, lower symmetries are possible. Figure $6 a-c$ show 3 possible reciprocal latties, whose superposition with a second lattice rotated by $90^{\circ}$ yields the observed diffraction pattern, as shown in Figure 4. Let us first consider the sharp reflections only which define an averaged lattice according to (1a) and Table 1. The first example (Fig. 6a) shows a strange extinction rule which cannot be explained in terms of one of the well known two-dimensional translation groups. These extinctions can only be interpreted by assuming further domains of lower translation symmetry; this more complicated case should be considered if the two remaining examples in Fig. 6 do not yield a satisfactory solution of the problem. Obviously, the sharp reflections shown in Fig. $6 \mathrm{~b}$, c give a $(2 \times 1)$ and $(1 \times 2)$ averaged structure ( $a=$ axis parallel to the diffuse streaks). Both patterns are different, but one of them (in Fig. $6 \mathrm{~b}$ ) looks very simple, because the reciprocal cell of the sharp reflections is centred by a diffuse one. The corresponding cell of this crystal is a centred $(4 \times 2)$-structure (orthogonal setting). The solution given in Fig. $6 \mathrm{c}$ again shows a $4 \times 2$ reciprocal cell but with an extinction rule that all beams $h^{\prime}=2$ (mod. 4) are missing and reflections $h^{\prime}=2 n+1$ are absent if $k^{\prime}=2 n$. It has been shown by the author [11] that a similar extinction rule is realized in the case of $\mathrm{SiC}$. Consequently, we are not entitled to reject this solution, but it looks complicated and should be postponed until the model in Fig. $6 \mathrm{~b}$ fails to be successful. From a structural point of view it is evident that the ordered chain is parallel to the Ge-Ge (Si-Si) chain, discussed in the dimer model by Levine [12] following one of the [110]-directions of the bulk crystal. Since the two models have this structural element in common, the 4-fold super-period is vertical to the chains. The sharpness of the corresponding superstructure reflections is nothing else but an order-disorder process of the anti-phase domains. The fact that this ordering process is influenced by twin-domains, lattice defects and impurities, may explain the different results reported by various authors.

Now we can show that none of the proposed models fully agrees with the diffuse diffraction data. If we assume that the chain parallel to $\boldsymbol{b}^{\prime}$ causes the diffuse streak $\left(\| a^{*}\right)$, we also need a doubled

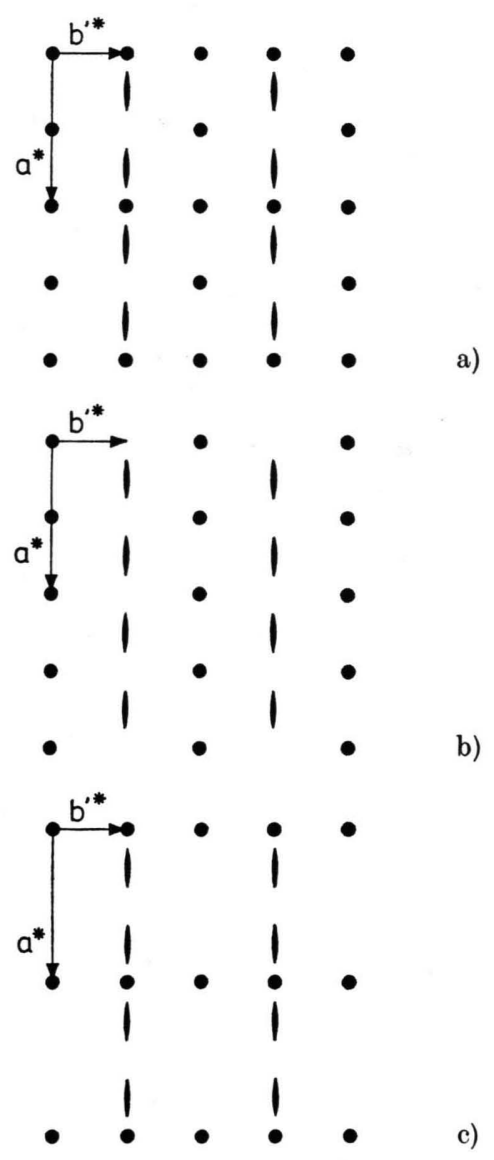

Fig. 6. Possible solutions for superposition of the diffraction patterns of twin domains through rotation by $90^{\circ}:$ a) $(2 \times 2)$ averaged structure; b) $(2 \times 1)$ averaged structure; c) $(1 \times 2)$ averaged structure. 
$a$-axis vertical to the chain direction. Consequently two different chains parallel to $\boldsymbol{b}$ having different structures, should be realized or the chain itself consists of two chains having different structures. This double row pairing should be the first ordering process since no diffuseness of $h=2 n+1$ in the $(2 \times 1)$-structure could be observed so far. Neither the Levine model with the better agreement with LEED-intensities - as shown by Appelbaum and Hamann [13] - nor the Seiwatz model [14] cope with this condition. This is also true for the model given by Poppendieck, Ngoc and Webb [15] although it contains two directions of ordering. Therefore a complete new structure determination should be started in order to explain all experimental observations, including disorder phenomena which may give information on important structural changes. Recently Chadi [16], [17], Ihm Cohen and Chadi [18], and Verwoerd [19] have discussed other models for the reconstruction of the (001)surface based on a theoretical calculation of dangling bonds. Their results seem to be supported by angle-resolved ultraviolet photoelectron spectroscopy published by Uhrberg, Hannsson, Nicholls, and Flodström [20]. All arguments given by these authors are convincing and the author agrees with the suggestion that structural models of lower symmetry have to be used to explain all experimental observations. As has been pointed out in Chapt. 3, twin domains cannot be avoided in this case. Consequently LEED-structure analysis becomes difficult, because disorder parameters have to be determined. This is stressed by the observation of diffuse scattering in the diffraction patterns of (001)-surfaces of $\mathrm{Si}$, and $\mathrm{Ge}$ as well. In contrast to the various models published so far, there is no principical difference between the $(2 \times 1)$ - and
$(4 \times 2)$-structure, which are correlated by an orderdisorder transition of the same structural unit. Obviously new experimental data are necessary to solve this complicated problem.

\section{Conclusions}

It is dangerous to start structure determinations of reconstructed surfaces without a preceding detailed study of the diffuse reflections using high precision diffraction patterns for the measurement of the diffuse background scattering and the accurate line profiles as well. They may indicate clearly as to whether an uncorrected measurement of integrated intensities will lead to a good agreement between observed and calculated intensities. Unfortunately structural papers describing reconstructions of lower point group symmetries often do not discuss a possible error caused by twin domains, which have to be considered if the diffraction pattern of the reconstructured surface shows a higher symmetry than realized in the suggested model. The assumption that large domain sizes admit an incoherent superposition of the diffraction patterns of the symmetrically equivalent individuals is incorrect. Since the bulk causes long range order of the few possible positions of domains, interference effects cannot be avoided even between larger domains. In the case of small domains a solution can be found by separating sharp reflections from diffuse scattering, but in this case all disorder parameters have to be determined.

The author thanks Drs. W. Moritz and D. Wolf for discussion of the complicated subject. This project has been supported within the Sonderforschungsbereich 128 by the Deutsche Forschungsgemeinschaft.

\section{Appendix}

\section{CEC-calculation of Disordered Surfaces (Two Dimensional Case)}

As defined in Chapt. $3, \boldsymbol{F}_{\boldsymbol{v}}\left(\boldsymbol{k}, \boldsymbol{k}^{\prime}\right)$ represents the contribution of the $\boldsymbol{v}$-th configuration of the central chain to the electrons scattered into the direction $\boldsymbol{k}\left(\boldsymbol{k}^{\prime}=\right.$ direction of incidence). The configuration $\boldsymbol{v}$ is formed by the central chain and a certain number of neighbours. In the zeroth approximation no neighbours, in the first approximation nearest neighbours are taken into account and so on. Consequently the number of possible configurations of a given central chain is $n^{z}$, if $n$ types of chains and $z$ neighbours have to be considered.

For a statistical arrangement of chains without long range order each cell with its origin at $j_{1} \boldsymbol{a}+j_{2} \boldsymbol{b}$ has the same a priori-probability $p_{\nu}$ to be occupied by a central chain in the configuration $v, \boldsymbol{a}, \boldsymbol{b}$ are the 
basis vectors of the bulk parallel to the surface, $h, k$ the corresponding components of the reciprocal vectors $h \boldsymbol{a}^{*}+k \boldsymbol{b}^{*}$.

With these definitions the resulting wave function of the scattered electrons becomes

$$
A\left(\boldsymbol{k}, \boldsymbol{k}^{\prime}\right)=\sum_{j_{1}=1}^{N_{\mathrm{a}}} \sum_{j_{2}=1}^{N_{\mathrm{b}}} F_{j_{1} j_{2}}\left(\boldsymbol{k}, \boldsymbol{k}^{\prime}\right) \exp \left\{2 \pi i\left(j_{1} h+j_{2} k\right)\right\},
$$

where $N_{\mathrm{a}}, N_{\mathrm{b}}$ are the numbers of unit cells in the corresponding directions. The indices $j_{1}, j_{2}$ shall indicate that in the cell $j_{1}, j_{2}$ one of the possible configurations $v$ is realized. Using ( ) as an abbreviation for $\left(\boldsymbol{k}, \boldsymbol{k}^{\prime}\right)$, we get the following equation for the intensity $I\left(k, k^{\prime}\right)$ :

$$
I()=|A()|^{2}=\sum_{j_{1}, j_{1}{ }^{\prime}=1}^{N_{\mathrm{a}}} \sum_{j_{2}, j_{j^{\prime}}=1}^{N_{\mathrm{b}}} F_{j_{1} j_{2}}() F_{j_{1} j_{j^{\prime}}}^{*}() \exp \left\{2 \pi i\left[\left(j_{1}-j_{1}{ }^{\prime}\right) h+\left(j_{2}-j_{2}{ }^{\prime}\right) k\right]\right\} .
$$

Introducing the new indices of summation

$$
m_{1}=j_{1}{ }^{\prime}-j_{1}, \quad m_{2}=j_{2}{ }^{\prime}-j_{2}
$$

the last equation transforms to

$$
I()=\sum_{j_{1}=1}^{N_{\mathrm{a}}-\left|m_{1}\right|} \sum_{j_{2}=1}^{N_{\mathrm{b}}-\left|m_{2}\right|} \sum_{m_{1}=-\left(N_{\mathrm{a}}-1\right)}^{+\left(N_{\mathrm{a}}-1\right)} \sum_{m_{2}=-\left(N_{\mathrm{b}}-1\right)}^{+\left(N_{\mathrm{b}}-1\right)} F_{j_{1} j_{2}}() F_{j_{1}+m_{1}, j_{2}+m_{2}}^{*}() \exp \left\{-2 \pi i\left(h m_{1}+k m_{2}\right)\right\} .
$$

The summation over $j_{1}, j_{2}$ may be performed by defining the a posteriori-probabilities

$$
p_{v \nu^{\prime}}\left(m_{1}, m_{2}\right) \text {. }
$$

They determine the probability that the cell at the distance $m_{1} \boldsymbol{a}+m_{2} \boldsymbol{b}$ apart from a given cell is found in the configuration $v^{\prime}$, if in the original unit cell the configuration $v$ was realized (correlation function). With this definition, and the a priori-probability $p_{v}$ already introduced we get

$$
I()=\sum_{m_{1}, m_{2}}\left(N_{\mathrm{a}}-\left|m_{1}\right|\right)\left(N_{\mathrm{b}}-\left|m_{2}\right|\right) \sum_{\nu, \nu^{\prime}} p_{\nu} p_{\nu \bar{\nu}^{\prime}}\left(m_{1}, m_{2}\right) F_{\nu}() F_{\nu^{\prime}}^{*}() \exp \left\{-2 \pi i\left(h m_{1}+k m_{2}\right)\right\} .
$$

In any statistical distribution without long range order the following relationship is valid:

$$
p_{v, v^{\prime}}\left(m_{1}, m_{2}\right) \approx p_{v}, \quad \text { if } m_{1}, m_{2}>n \quad \text { (vanishing correlation). }
$$

Hence (A3) may be seperated into two sums

$$
\begin{aligned}
I() & =\sum_{m_{1}= \pm(n+1)}^{ \pm\left(N_{\mathrm{a}}-1\right)} \sum_{m_{2}= \pm(n+1)}^{ \pm\left(N_{\mathrm{b}}-1\right)}\left(N_{\mathrm{a}}-\left|m_{1}\right|\right)\left(N_{\mathrm{b}}-\left|m_{2}\right|\right) \sum_{\nu, \nu^{\prime}} p_{\nu} p_{\nu^{\prime}} F_{\nu}() F_{\nu^{\prime}}^{*}() \exp \left\{-2 \pi i\left(h m_{1}+k m_{2}\right)\right\} \\
& +\sum_{m_{1}=-n}^{+n} \sum_{m_{2}=-n}^{+n}\left(N_{\mathrm{a}}-\left|m_{1}\right|\right)\left(N_{\mathrm{b}}-\left|m_{2}\right|\right) \sum_{\nu, \nu^{\prime}} p_{\nu} p_{\nu \nu^{\prime}}\left(m_{1}, m_{2}\right) F_{\nu}() F_{v^{\prime}}^{*}() \exp \left\{-2 \pi i\left(h m_{1}+k m_{2}\right)\right\} .
\end{aligned}
$$

Now we complete the sums over $m_{1}, m_{2}$ of the first term with the missing elements

$$
\sum_{m_{1}=-n}^{+n} \sum_{m_{2}=-n}^{+n}\left(N-\left|m_{1}\right|\right)\left(N-\left|m_{2}\right|\right) p_{\nu} p_{v^{\prime}} F_{\nu}() F_{\nu^{\prime}}^{*}() \exp \left\{-2 \pi i\left(h m_{1}+k m_{2}\right)\right\}
$$

and subtract them from the second term. Then we get

$$
\begin{aligned}
I()= & \sum_{m_{1}=-\left(N_{\mathrm{a}}-1\right)}^{+\left(N_{\mathrm{a}}-1\right)} \sum_{m_{2}=-\left(N_{\mathrm{b}}-1\right)}^{+\left(N_{\mathrm{b}}-1\right)}\left(N_{\mathrm{a}}-\left|m_{1}\right|\right)\left(N_{\mathrm{b}}-\left|m_{2}\right|\right) \exp \left\{-2 \pi i\left(m_{1} h+m_{2} k\right)\right\} \sum_{\nu} p_{v} F_{v}() \sum_{v^{\prime}} p_{v^{\prime}} F_{v^{\prime}}^{*}() \\
& +\sum_{m_{1}, m_{2}=-(n-1)}^{+(n-1)} N_{\mathrm{a}} N_{\mathrm{b}} \sum_{v^{\prime} v^{\prime}} p_{v}\left[p_{v v^{\prime}}\left(m_{1}, m_{2}\right)-p_{v^{\prime}}\right] F_{v}() F_{v^{\prime}}^{*}() \exp \left\{-2 \pi i\left(m_{1} h+m_{2} k\right)\right\} \\
= & I_{\mathrm{s}}()+I_{\mathrm{d}}(), \quad\left(I_{\mathrm{s}}=\text { sharp reflections, } \quad I_{\mathrm{d}}=\text { diffuse scattering }\right) .
\end{aligned}
$$


Since $\left|m_{1}\right|,\left|m_{2}\right| \leqq n,\left|m_{1}\right|\left|m_{2}\right|$ may be neglected against $N_{\mathrm{a}}, N_{\mathrm{a}}$ in the second term, the first term in (A4) can be evaluated and becomes (periodic avaraged lattice!)

$$
I_{\mathrm{s}}()=\frac{\sin ^{2} \pi N_{\mathrm{a}} h}{\sin ^{2} \pi h} \frac{\sin ^{2} \pi N_{\mathrm{b}} k}{\sin ^{2} \pi k}|\overline{F()}|^{2}=R^{2}|\overline{F()}|^{2} .
$$

The second term in (A4) can be rewritten by remembering that

$$
\begin{gathered}
p_{v v^{\prime}}(0,0)=\delta_{v v^{\prime}} \quad\left(\delta_{v v^{\prime}}=1 \quad \text { if } v=v^{\prime},=0 \text { otherwise }\right), \\
\begin{array}{c}
I_{\mathrm{d}}()=N_{\mathrm{a}} N_{\mathrm{b}}\left\{\left(\left.\left|\overline{\left.F()\right|^{2}}-\right| \overline{F()}\right|^{2}\right)+\sum_{m_{1}, m_{2}=0} \sum_{v, v^{\prime}}\left[p_{v}\left(p_{v v^{\prime}}\left(m_{1}, m_{2}\right)-p_{v}\right)\right]\right. \\
\left.\cdot F_{v}() F_{v^{\prime}}^{*}() \exp \left\{-2 \pi i\left(m_{1} h+m_{2} k\right)\right\}\right\} .
\end{array}
\end{gathered}
$$

It may easily be shown that the second term is small when compared with first one, if a random distribution is realized. If short range order cannot be neglected, (A6) should be transformed to

$$
\begin{aligned}
I_{\mathrm{d}}()= & N_{\mathrm{a}} N_{\mathrm{b}}\left[\left(\overline{|F()|^{2}}-|\overline{F() \mid}|^{2}\right)\right] \sum_{m_{1}, m_{2}=-n}^{+n} \sum_{\nu, v^{\prime}} \frac{p_{v}\left[p_{v v^{\prime}}\left(m_{1} m_{2}\right)-p_{v^{\prime}}\right]}{\left(\overline{\left|F^{\prime}()\right|^{2}}-|\bar{F}()|^{2}\right)} \\
& \cdot \boldsymbol{F}_{\boldsymbol{v}}() F_{v^{\prime}}^{*}() \exp \left\{-2 \pi i\left(m_{1} h+m_{2} k\right)\right\} .
\end{aligned}
$$

Since $p_{v v^{\prime}}\left(m_{1}, m_{2}\right)-p_{v^{\prime}}$ approaches zero with increasing $m_{1}, m_{2}$ and the term $m_{1}=m_{2}=0$ of the sum is unity, (A7) describes diffuse reflections modulated with the factor $N_{\mathrm{a}} N_{\mathrm{b}}\left[\overline{\left[\left.F\right|^{2}\right.}-|\bar{F}|^{2}\right]$.

\section{One-dimensional case}

Let us assume that the crystal surface be perfectly ordered in the $\boldsymbol{b}$-direction, then $F_{j_{1}, j_{2}}()$ in (A1) may be replaced by $F_{j_{1}}\left(\right.$ ), (the cell occupation is no longer dependent on $j_{2}$ !).

The summation over $j_{2}$ may be performed, and we get

$$
A()=\frac{\sin \pi N_{\mathrm{b}} k}{\sin \pi k} \sum_{j_{1}=1}^{N_{\mathrm{a}}} F_{j_{1}}() \exp \left\{2 \pi i j_{1} h\right\}=R_{\mathrm{b}} \sum_{j_{1}} F_{j_{1}}() \exp \left\{2 \pi i j_{1} h\right\} .
$$

Now, the whole procedure described above may be repeated, and ends up with similar formulae, where summations over $j_{2}, m_{2}$ do not appear any longer. Equation (A5) remains unaltered, (A6) changes to

$$
I_{\mathrm{d}}()=N_{\mathrm{a}} R_{\mathrm{b}^{2}}\left\{\left.\left|\overline{\left.F()\right|^{2}}-\right| \bar{F}()\right|^{2}+\sum_{m_{1} \neq 0}^{n} \sum_{\nu, v^{\prime}} p_{v}\left[p_{v v^{\prime}}\left(m_{1}\right)-p_{v^{\prime}}\right] \exp \left\{-2 \pi i m_{1} h\right\}\right\} .
$$

In order to elucidate the theory outlined above, let us discuss the simple example of two kinds of chains obeying a statistical distribution with a priori-probabilities $p_{1}$ and $p_{2}=1-p_{1}$. In the zeroth approximation (multiple scattering of neighbours is taken into account by averaging) the contributions to scattering are

$$
F_{1}^{(0)}\left(\boldsymbol{k}, \boldsymbol{k}^{\prime}\right), \quad F_{2}^{(0)}\left(\boldsymbol{k}, \boldsymbol{k}^{\prime}\right)
$$

Hence we get the following expressions for $|\bar{F}|^{2}$ and $\overline{|F|^{2}}-|\bar{F}|^{2}$ entering (A5) and (A9):

$$
\begin{aligned}
& |\bar{F}()|^{2}=\left|p_{1} F_{1}^{(0)}()+\left(1-p_{1}\right) F_{2}^{(0)}()\right|^{2} \\
& |\overline{F(}()|^{2}-|\overline{F()}|^{2}=p_{1}\left(1-p_{1}\right)\left|F_{1}^{(0)}()-F_{2}^{(0)}()\right|^{2} .
\end{aligned}
$$

For a random distribution of chains we have no correlations between neighbouring chains, consequently $p_{v v^{\prime}}(1)=p_{v^{\prime}}$, and it may be shown by induction that all $p_{v v^{\prime}}\left(m_{1}\right)=p_{v^{\prime}}$ as long as $m_{1} \neq 0$.

Consequently, the upper limit of summation in (A9) is $n=0$, and the second term vanishes. Disregarding the fact that $F_{v}^{(0)}\left(\boldsymbol{k}, \boldsymbol{k}^{\prime}\right)$ may contain contributions to multiple scattering originating from atoms within the chain and the averaged surrounding, the final result is equivalent to a kinematical calculation. Hence we may call the zeroth approximation the "pseudo-kinematical approximation". In the first approxima- 
tion we have to include the two nearest neighbours. Hence, the two types of chains occur in 4 different configurations:
type 1:
1. $\{111\}$,
2. $\{112)$,
3. $\{211\}$,
4. $\{212\}$,
type 2:
5. $\{222\}$,
6. $\{221\}$,
7. $\{122\}$,
8. $\{121\}$,

e.g. the sequence of 10 chains

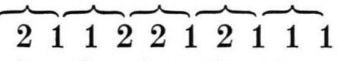

corresponds to the sequence of the following 10 configurations:

$\{.21\},\{211\},\{112\},\{122\},\{221\},\{212\},\{121\},\{211\},\{111\},\{11$.$\} .$

Two adjacent configurations have the last two and the first two types of chains in common, and the central symbol refers to the central chain. In the first approximation we have to introduce 8 different $F_{\nu}^{(1)}()$ :

$$
F_{v}^{(1)}()=F_{v}^{(0)}()+\Delta F_{v}(), \quad v=1, \ldots, 8 .
$$

The following new a priori-probabilities $p_{v}{ }^{\prime}$ must be defined for the eight configurations

$$
\begin{aligned}
& \{111\}: p_{1}^{\prime}=p_{1} p_{11}(+1) p_{11}(-1) \\
& \{211\}: p_{2}^{\prime}=p_{1} p_{11}(+1) p_{12}(-1) \\
& \ldots \ldots \ldots \ldots \ldots \ldots \ldots \\
& \{121\}: p_{8}^{\prime}=p_{2} p_{21}(+1) p_{21}(-1) . \\
& |\overline{F()}|^{2} \text { is now given by } \\
& |\overline{F()}|^{2}=\left|\sum_{\nu=1}^{4} p_{\nu}^{\prime}\left[F_{1}^{\prime}()+\Delta F_{v}()\right]+\sum_{\nu=5}^{8} p_{\nu}^{\prime}\left[F_{2}^{0}()+\Delta F_{v}()\right]\right|^{2} \\
& \quad=\left|\left[p_{1} F_{1}^{(0)}()+\left(1-p_{1}\right) F_{2}^{(0)}()\right]+\sum_{\nu=1}^{8} p_{\nu}^{\prime} \Delta F_{\nu}()\right|^{2} .
\end{aligned}
$$

The term in square brackets corresponds to the result received for the zeroth approximation given in (A1), but it will be shown below that the sum (second term) is not small when compared with the preceding one.

In a similar way $\overline{\left|F^{2}\right|}-|\bar{F}|^{2}$ may be calculated, and the result may be expressed by two terms in a similar way:

$$
\overline{|F()|^{2}}-|\overline{F()}|^{2}=p_{1}\left(1-p_{1}\right)\left|F_{1}^{(0)}()-F_{2}^{(0)}()\right|^{2}+\text { correcting terms for multiple scattering. }
$$

Again the correcting terms of the first approximation are not small. Another important point should be stressed: In the random distribution the relation

$$
p_{v v^{\prime}}\left(m_{1}\right)=p_{v^{\prime}}
$$

is no longer valid for $1 \leqq m_{1} \leqq 2$. Hence, the sum in (A9) does not vanish, as it was true in the zeroth approximation. But the correction given by the sum is small against the first term in (A9) and shall not be considered here.

\section{Approximate Calculation of $\Delta F_{i}$}

Since LEED-structure determinations of ordered crystal surfaces may be carried out with a fair agreement between calculated and observed intensities, an estimate of the $\Delta F_{i}$ entering the first approximation may be given. We calculate the diffraction of 8 structures containing the $8 \Delta F_{i}$ needed. The most simple structures are:
1. $(1 \times 1)$-structure,
$11 \overline{1} \ldots$,
$\{111\} \overline{\{111\}} \ldots$,
2. $(1 \times 1)$-structure,
$22 \overline{2} \ldots$,
$\{222\} \overline{\{222\}} \ldots$, 


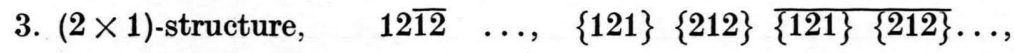

4. $(3 \times 1)$-structure, $112 \overline{112} \ldots, \quad\{211\}\{112\}\{121\} \ldots$,

5. $(3 \times 1)$-structure, $221 \overline{221} \ldots, \quad \overline{\{122\}\{221\}\{212\}} \ldots$,

6. $(4 \times 1)$-structure, $1112 \overline{1112} \ldots, \overline{\{211\}\{111\}\{112(\{121\} \ldots,}$

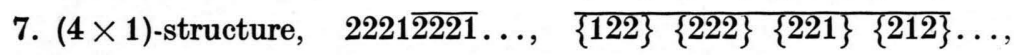

8. $(4 \times 1)$-structure, $1122 \overline{1122} \ldots, \overline{\{211\}}\{\overline{112\}}\{122\}\{221\} \ldots$

The symbols in curly brackets represent the periodic sequence of cluster embedded chains. Following conventional methods of LEED-structure determination eight different patterns $F_{i}^{t}\left(\boldsymbol{k}, \boldsymbol{k}^{\prime}\right)$ may be calculated. On the other hand another computation may be done with the aid of $F_{v}^{(1)}\left(\boldsymbol{k}, \boldsymbol{k}^{\prime}\right)$ according to the first approximation given in (A1), evaluated for the structures described above.

Using the definitions given in (A12, A13), we get

$$
\begin{aligned}
& F_{1}()=F_{1}^{(1)} R, \quad F_{2}()=F_{5}^{(1)}() R, \quad F_{3}()=\frac{1}{2}\left[F_{8}^{(1)}()+e^{2 \pi i h} F_{4}()\right] R, \\
& F_{4}()=\frac{1}{3}\left[F_{3}^{(1)}()+F_{2}^{(1)}() e^{2 \pi i h}+F_{8}^{(1)}() e^{4 \pi i h}\right] R, \quad \text { etc. }
\end{aligned}
$$

where $R=R_{\mathrm{a}} R_{\mathrm{b}}$, and the factors $1 / 2$ etc. result from the reduced number of unit cells for superstructures. In this way we get 8 equations with the eight unknown variables $F_{v}^{(1)}\left(k, k^{\prime}\right)$. The $F_{i}\left(k, k^{\prime}\right)$ should be equal to $F_{i}^{t}\left(\boldsymbol{k}, \boldsymbol{k}^{\prime}\right)$ if the approximation is accurate.

Consequently

$$
F_{i}^{\mathrm{t}}\left(\boldsymbol{k}, \boldsymbol{k}^{\prime}\right) \approx F_{i}\left(\boldsymbol{k}, \boldsymbol{k}^{\prime}\right) .
$$

The error of this equation is mainly due to the fact that in a disordered surface the configuartions are embedded in a statistical surrounding. This is no longer true for the strictly ordered surface. Now (A16) may be solved, if all $F_{i}^{\mathrm{t}}\left(\boldsymbol{k}, \boldsymbol{k}^{\prime}\right), \boldsymbol{F}_{i}\left(\boldsymbol{k}, \boldsymbol{k}^{\prime}\right)$ are independent. Since the $F_{i}^{\mathrm{t}}\left(\boldsymbol{k}, \boldsymbol{k}^{\prime}\right)$ result from different structures, the $F_{v}^{(1)}\left(k, k^{\prime}\right)$ received should all be different. According to (A13) we have

$$
F_{v}^{(1)}()-F_{v^{\prime}}^{(1)}()=\Delta F_{v}()-\Delta F_{v^{\prime}}() \text {. }
$$

We may now conclude from (A16) and (A17) that the $\Delta F()$ should have the same order of magnitude, as it holds for the differences of $F_{i}^{t}\left(\boldsymbol{k}, \boldsymbol{k}^{\prime}\right)$. Since completely different structures are involved, the $\Delta \boldsymbol{F}_{\boldsymbol{i}}\left(\boldsymbol{k}, \boldsymbol{k}^{\prime}\right)$ cannot be small for all $\boldsymbol{k}, \boldsymbol{k}^{\prime}$. The reader might be puzzled about the fact that different beams are calculated for the various structures. All structures involved contribute to $k=$ integer, e.g. half order beams are not calculated for all of them. But it may easily be shown that this difficulty will be resolved by only choosing structures contributing to the beams wanted.

\section{Lamellar Anti-phase Domains}

In the preceding sections the disorder phenomena of a $(2 \times 1)$-structure has been discussed with the $\boldsymbol{b}$-axis as direction of the lamellae. This means that there are strong correlations in this direction, and a strong propagation of order is involved. If interactions of nearest neighbours are predominant, three different ordered structures may be realized, namely

$$
111 \overline{1} \ldots, 222 \overline{2} \ldots, 12 \overline{12} \ldots,
$$

where the latter is equivalent to $21 \overline{21} \ldots$, differing by a displacement only (vertical to the direction of lamellae in disordered structures). Apparently there is another possibility of generating anti-phase domains. In accordance with the discussion of the model in Chapt. 3 we assume that the propagation of order is predominant in the direction of superstructure formation.

Then the super-period is formed parallel to the direction of lamellae $(b)$, and a $(1 \times 2)$-structure is realized. Consequently in the zeroth approximation the row of cluster embedded chains parallel to $b$ consists of two alternating types 1, 2. In the first approximation there is no alteration in the $b$-direction, but different neighbouring rows of chains (a-direction) must be taken into account (Figure 2 ). They have 
the same superstructure, but may be displaced by $\boldsymbol{b}$ (anti-phase vector parallel to the direction of lamellae). Taking the central chain as representative for the whole row, as shown by the arrow in Fig. 2a, the symbol $\{111\}$ represents a cluster embedded chain in the 1 position surrounded by two other chains in the same position. Correspondingly $\{222\}$ has the analogous meaning for a central chain in position 2 . On account of these definitions an interchange of 1 and 2 is equivalent to a displacement by $\boldsymbol{b}$, consequently $\{111\}$ is equivalent to $\{222\},\{112\}$ to $\{221\}$ etc. On account of this equivalence we have (compare Sect. 2 of the Appendix) $p_{1}=p_{2}=1 / 2$. For the same reason the relations

$$
p_{11}(1)=p_{11}(-1)=p_{22}(1)=p_{22}(-1)=\alpha \quad \text { and } \quad p_{12}(1)=p_{12}(-1)=p_{21}(1)=p_{21}(-1)=1-\alpha
$$

are valid. Since the ordered rows parallel to $\boldsymbol{b}$ have the alternating structure with the unit cell vector $\boldsymbol{b}^{\prime}=2 \boldsymbol{b}, k^{\prime}=2 k$, the summation of contributions to scattering leads to a Laue-factor

$$
R_{\mathrm{b}}{ }^{\prime}=\frac{\sin \pi N_{\mathrm{b}} k^{\prime}}{\sin \pi k^{\prime}} \text {. }
$$

Readers, who want to stick to the old unit cell, should remember that $k^{\prime}=2 n$ (even) corresponds to $k=n$ (integral order beams) and correspondingly $k^{\prime}=2 n+1$ (odd) to $k=\frac{1}{2}(2 n+1)$ (half order beams). Now, the displacement vector between equivalent clusters is $\boldsymbol{b}^{\prime} / 2(=\boldsymbol{b})$, consequently a phase factor $e^{\pi i k^{\prime}}$ has to be introduced for equivalent clusters (see Table 1 in Chapter 3). Now $\overline{\boldsymbol{F}\left(\boldsymbol{k}, \boldsymbol{k}^{\prime}\right)}$ may easily be calculated $\left[F_{1}^{(0)}()=F_{2}^{(0)}()=F_{0}()\right]$

$$
\begin{gathered}
\overline{F()}=\frac{1}{2}\left(1+e^{\pi i k^{\prime}}\right)\left\{(1-\alpha)^{2}\left[F_{0}()+\Delta F_{1}()\right]+\alpha(1-\alpha)\left[2 F_{0}()+\Delta F_{2}()+\Delta F_{3}()\right]\right. \\
\left.+\alpha^{2}\left[F_{0}()+\Delta F_{4}()\right]\right\} .
\end{gathered}
$$

For $k^{\prime}=$ odd $\bar{F}$ in (A18) vanishes.

Computation of $|\bar{F}|^{2}$ according (A18) yields (2a) in Chapter 3. In a similar manner $\overline{|F|^{2}}$ may be given:

$$
\begin{aligned}
\overline{|F()|^{2}}= & \left(1-\alpha^{2}\right)\left|F_{0}()+\Delta F_{1}()\right|^{2}+\alpha(1-\alpha)\left[\left|F_{0}()+\Delta F_{2}\right|^{2}+\left|F_{0}()+\Delta F_{3}()\right|^{2}\right] \\
& +\alpha^{2}\left|F_{0}()+\Delta F_{4}()\right|^{2} .
\end{aligned}
$$

Equation (A19) is valid for $k^{\prime}=$ even, and $k^{\prime}=$ odd as well. Its evaluation gives $(2 \mathrm{~b})$.

[1] I. R. Noonan and H. L. Davis, Proc. 4th Int. Conf. of Surf. Science, and the 3rd Europ. Conf. of Surf. Science 1, 100 (1980).

[2] E. Zanazzi and F. Jona, Surface Sci. 62, 61 (1977).

[3] H. Jagodzinski, W. Moritz, and D. Wolf, Surface Sci. 77, 233 (1978).

[4] H. Jagodzinski, Phys. stat. sol. (a) 49, 119 (1978).

[5] W. Moritz, H. Jagodzinski, and D. Wolf, Surface Sci. 77, 249 (1978).

[6] H. Jagodzinski and B. Penzkofer, Acta Cryst. A 37, 745 (1981).

[7] W. Adlhart, Acta Cryst. A 37, 794 (1981).

[8] B. Z. Olshanetsky, S. M. Repinsky, and A. Shklyaev, Surface Sci. 64, 224 (1977).

[9] J. J. Lander and J. Morrison, J. Appl. Phys. 33, 2089 (1962).

[10] F. Jona, H. D. Shih, D. W. Jepsen, and P. M. Marcus, Solid State Phys. 12, L 455 (1979).
[11] H. Jagodzinski, Kristallografiya, 16, 1235 (1971). Sov. Phys. Cryst. 16, 1081 (1972).

[12] J. P. Levine, Surface Sci. 34, 90 (1972).

[13] J. A. Appelbaum and D. R. Hamann, Surface Sci. 74, 21 (1978).

[14] R. Seiwatz, Surface Sci. 2, 473 (1964).

[15] T. D. Poppendieck, T. C. Ngoc, and M. B. Webb, Surface Sci. 75, 287 (1978).

[16] D. J. Chadi, Phys. Rev. Lett. 43, 43 (1979).

[17] D. J. Chadi, J. Phys. Soc. Japan Suppl. A 49, 1035 (1980).

[18] J. Ihm, M. L. Cohen, and P. J. Chadi, Phys. Rev. B 21, 4592 (1980).

[19] W. S. Verwoerd, Surface Sci. 99, 581 (1980).

[20] R. I. G. Uhrberg, G. V. Hansson, J. M. Nicholls, and S. A. Flodström, Phys. Rev. B 24, 4684 (1981). 